\title{
DESIGNING A NEW TYPE OF ELECTRIC MACHINERY CONTINUOUSLY VARIABLE TRANSMISSION COMBINED WITH MATIA/SIMULINK SIMULATION KINEMATICS MODEL BASED ON CATIA AND ADAMS
}

\author{
Ming-ming $W u^{1}$, Xing Wang ${ }^{1}$, Dinesh Surroop ${ }^{1}$, Kamalesh Prasad ${ }^{1}$ \\ ${ }^{1}$ Anhui Sanlian University, Heifei Anhui 230601, China
}

\begin{abstract}
Objective: In order to break through the limitations of traditional electric machinery transmission and strengthen the stability of electric machinery transmission. Method: This paper conducts the design mainly based on the CATIA and ADAMS and combined with matlab/simulink simulation kinematics model and configure the main motor and conveyor through different parameters. Results and analysis: The design in this paper has a good performance in terms of efficiency and acceleration performance. Conclusion: The characteristics of various transmission schemes designed in this paper are analyzed, including speed, power flow direction and efficiency characteristics; and the combinatory analysis of the characteristics of the two-period planetary transmission is conducted based on the one-part planetary gear.
\end{abstract}

Keywords: Matia/Simulink Simulation Kinematics Mode, CATIA, ADAMS.

\section{Introduction}

Automobile is a very popular transportation vehicle in modern society. In its structure, transmission is an important component of automobile power. However, the dynamic mechanical characteristics of the power source output of most modern automobiles can only satisfy the power demand through the deceleration and the torque increase of the transmission instead of directly acting on the automobile itself. Under this premise, because one more step is added, all aspects of the automobile's performance, such as speed adjustment and speed increase will be affected and the speed change and torque increase may lead to a shortened service life of the automobile. Therefore, how to directly apply the power of the transmission to the action of the automobile is a problem that is highly regarded in the related field.

Based on this problem, a new type of electric machinery continuously variable transmission is designed based on the CATIA and ADAMS combined with mat lab/simulink kinematics simulation model.

Theoretically, this transmission can break through the limitations of the traditional transmission operation and ignore the deceleration and increase the torque but directly apply the power to the action of the automobile, thereby avoiding the disadvantages of impaired automobile performance and shortened service life.
The main design idea of this paper is: the dual motor is used as the power source and the main motor $\mathrm{Ml}$ mainly provides the driving force.

The speed-regulating motor M2 plays the role of regulating the output speed.

Finally, through the actual verification, it is found that the designed transmission has the advantages of relatively large transmission, wide range of speed regulation and high system flexibility.

\section{Literature review}

At present, with the shortage of energy, the demand for environmental protection is increasing.

The traditional automotive industry has been greatly affected. The development and utilization of new energy has become a major trend in the coming period of time. In this environment, electric vehicles have received more and more attention due to their superior energy conservation and environmental protection. As a bridge between the matching power source and the vehicle, the rational design of the transmission parameters can effectively enhance the vehicle's dynamic performance and economic performance. It also reduces the driving conditions for motor performance requirements. In the vehicle transmission system, the transmission is a very important component.

In the aspect of power performance matching, the continuously variable transmission can more fully 
match the power source and the vehicle than the conventional stepped transmission.

The transmission is an important part of the automotive power train. At present, the mechanical properties of most automotive power sources (including engines and motors) cannot be directly used for vehicle driving. It generally requires transmission deceleration to meet actual driving needs. The related research on the transmission is related to the transmission function of the transmission and the driving wheel. Its functions generally include adjusting speed, transmitting power and changing power. The pros and cons of the transmission function are closely related to the dynamics of the vehicle and the quality of the economy, and may even affect the life of the vehicle (Cheng et al., 2015).

According to the transmission ratio, the transmission can generally be categorized into two major categories: stepless transmission and continuously variable transmission. The transmission ratio of Continuously Variable Transmission (CVT) can be continuously changed within a certain interval. The CVT enables continuous adjustment of the speed so that the power source matches the entire vehicle to the best performance. It not only guarantees the economy and power of the vehicle, but also improves the operator's driving feel and operating comfort.

According to the control and transmission methods, the CVT can be categorized into three types: mechanical, fluid, and electric. In general, conventional stepless transmissions use only one of the above forms of power transmission. Composite induction drive technology was studied.

With the improvement of the transmission system requirements and the advancement of technology, a variety of power transmission forms of composite continuously variable transmission technology is gradually developed and has been applied (Druant et al., 2016).

With the ever-decreasing storage of nonrenewable resources such as petroleum and the growing popularity of the concept of green environmental protection, the major products of the automotive industry are gradually transforming into new energy vehicles. Today, the main new energy vehicles include pure electric vehicles and hybrid cars. In such vehicles, motors are used as power sources (Liu et al., 2016). As mentioned before, the power performance that can be provided only for the speed regulation of the motor is limited.

In particular, under conditions where the load resistance is large, such as climbing, the current motor manufacturing level is still unable to meet the torque. Simply increasing the motor power will result in an excessively large volume, which cannot be applied to the vehicle. Since there is no subsequent transmission, the efficiency of the motor cannot be guaranteed during speed regulation, that is, the system transmission efficiency will be lower in certain speed ranges. In order to solve the problem of the lack of power of the simple electric drive, most hybrid vehicles adopt the way to combine the power provided by the power source via the bus.

The motor and the engine are respectively connected with the power elements of the planetary row. With the planetary gear mechanism, it is easy to achieve a continuous change of the transmission ratio and an increase in the output torque. It can improve the matching of the dynamic performance of two power sources and improve the efficiency of energy utilization (Petković et al., 2014). Toyota's hybrid car started very early. It is the best technology automobile company in the world at this stage.

The Prius transmission system of its hybrid production car uses the planetary platoon coupling mechanism. There is no complicated gear shaft in this drive train. Only one planetary group has a simple structure. Without a torque converter, the power source is directly connected to the transmission and the efficiency is extremely high. In recent years, hybrid technology has developed rapidly (Shamshirband et al., 2014).

In terms of the transmission system, the dual clutch transmission was successfully applied to the G6, Speedy, Qin and other models. BYD's transmission system uses a parallel mode. The performance is good. The two-speed clutch gearbox connects the engine and the motor. It is highly dynamic, comfortable, and quiet (Sulaiman et al., 2015).

The start of pure electric vehicles is much later than that of hybrid vehicles, and its penetration rate is currently low. In pure electric vehicles, Tesla is currently the most technologically advanced in the world. Tesla-MODEL series electric vehicles use electric motor stepless transmission.

Based on the research of a stepless gear reducer, it was found that the motor was connected with the drive wheels to achieve the purpose of enhancing output power (Tyreas and Nikolakopoulos, 2016).

With the increasing scarcity of oil and other energy resources, environmental issues such as global warming continue to highlight, and "energy conservation and environmental protection" has become the main topic of today's social development. Traditional fuel vehicles have significant shortcomings in energy sources, energy efficiency, and environmental impact. The transition from traditional energy vehicles to new energy vehicles is imperative.

The electric power source of the electric vehicle adopts the on-board power supply, and the electric motor is used as a direct input device to replace the 
fuel engine. It has great advantages in terms of high energy efficiency and low environmental pollution.

This will be the focus of future development in the auto industry (Yang et al., 2016).

To sum up, the above research works are mainly focused on the research of continuously variable transmission of electric machinery from the stepless transmission technology.

Therefore, a new type of electromechanical continuously variable transmission based on CATIA, ADAMS and mat lab/simulink simulation kinematics models is studied. The stepless drive test stand for electrical machinery was built.

The designed CVT was tested under steady state conditions. Its operating data is collected and analyzed. The electric mechanical stepless transmission has stable stepless speed regulation characteristics and high system efficiency characteristics within its speed regulation range.
Due to the limitation of test conditions and time, the test verification of uniform operating conditions was performed only for electric mechanical stepless transmissions. In the follow-up work, special hardware and software development control shall be further conducted on the accelerated and steady operating conditions to complete the test verification under the corresponding working conditions.

\section{Method}

\subsection{Transmission scheme}

The two motors of the electric machinery continuously variable transmission can be connected with two connections of the three components of the planetary gear as the power input shaft, which include 6 different ways of combination, as is shown in Figure 1.

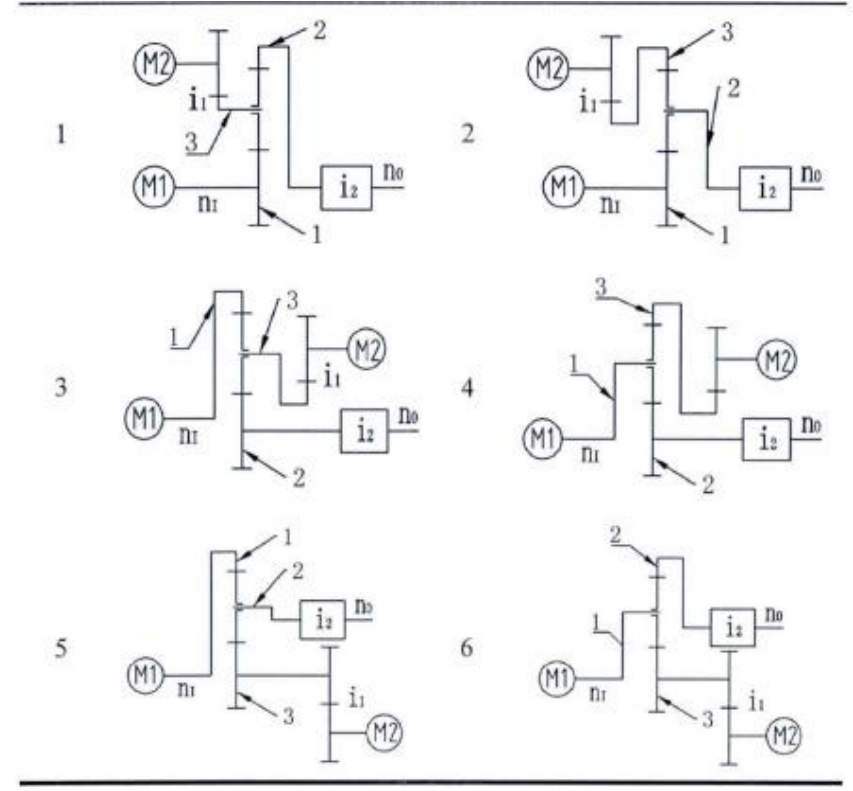

$\overline{\text { Figure 1: Schematic diagram of each transmission scheme }}$

In the figure, $\mathrm{nI}$ and $\mathrm{n} 0$ indicate the rotation speed corresponding to the input and output; the 1 , 2 and 3 shafts represent the input and output shafts; in order to ensure the power output to the wheels via the planetary gear, the planetary gear is connected to a first gear mechanism to reduce speed and increase the torque and the transmission ratio is i2; a first gear transmission device is added between the speed control motor M2 and the input shaft and the transmission ratio is $\mathrm{i} 1$.

\subsection{Characteristics of continuously variable transmission}

As mentioned before, the electric machinery continuously variable transmission realizes the continuously variable by adjusting the input speed of the two motors. When adjusting the speed of M1 and $\mathrm{M} 2$, the speed ratio e of the two motors will change accordingly.

The characteristics of continuously variable refer to the characteristics of the change of the speed ratio i between the input and output of the entire electric machinery continuously variable transmission system with the speed ratio e of the two motors.

After analyzing the scheme in Figure 1, three schemes of 3,5 , and 6 are selected and the speed characteristics curve of the three schemes are also obtained, as is shown in Figure 2, 3, and 4. 

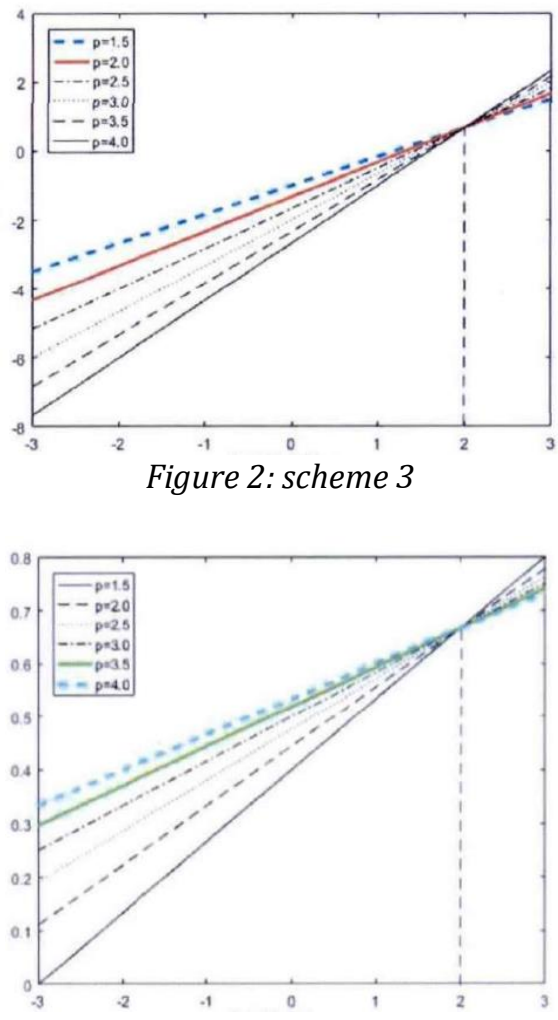

Figure 3: scheme 5

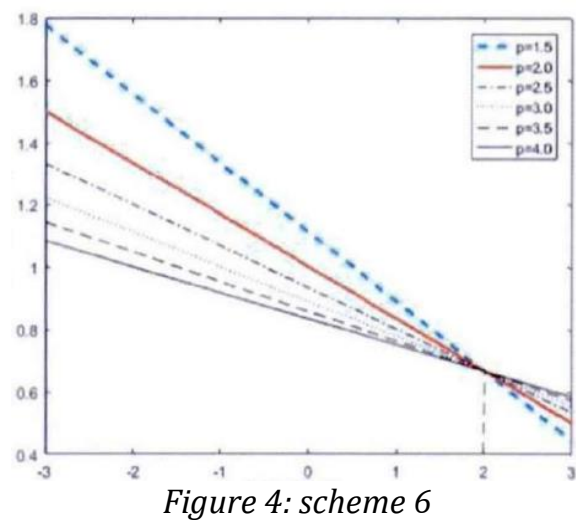

It can be seen from Figure 2, 3 and 4 that in the electric machinery continuously variable transmission studied in this paper, there is a linear correlation between the reverse speed ratio i' of the drive system and the motor speed ratio e, which is suitable for the automobile drive system and is easier to control.

Comparing the above three schemes, the reverse speed ratio i' of scheme 3 and 5 monotonically increases as the motor speed ratio e increases from 3 to 3 and the reverse speed ratio i' in scheme 6 monotonously decreases as the motor speed ratio e increases from -3 to 3 . This indicates that the rotation direction of the output from the planetary gear 2 shaft to the wheels via the transmission is variable, which means that these three schemes can realize forward and reverse driving.
In scheme 3, the reverse ratio is negative, representing that the output speed and input reversion can realize reverse driving while scheme 5 and 6 can realize forward driving. Therefore, the scheme 3 has relatively large range of speed regulation under the given parameter.

\subsection{Efficiency characteristics}

The electric machinery continuously variable transmission system uses the motor to directly drive the planetary gear.

To facilitate the study, the efficiency loss of the motor system is ignored That is to say, there is no power loss from the power input to the planetary gear in electric machinery continuously variable transmission system and the energy loss of the entire electric machinery continuously variable transmission system exists only in the mechanical transmission part.

This paper adopts a closed planetary gear transmission.

Because of the characteristics of the gear train itself, there will be an internal power cycle. From the analysis of the power split ratio of the speed regulating motor, it can be seen that in each scheme, the speed control motor M2 is not always used as a power source to input power to the system.

When $\mathrm{p}<0$, the speed-regulating motor M2 is used as the generator to consume the power from the planetary gear, namely the main motor M1 and in this case, there is inevitably an internal power consumption to decrease the transmission efficiency.

\subsection{Optimal design of electric machinery continuously variable transmission}

This paper will use a small electric automobile as the research object and design a suitable continuously variable transmission for its specific working conditions, which completes the power matching of the electric machinery continuously variable transmission and the motor.

Combined with various parameters of the automobile, it ensures relatively large transmission range and the transmission efficiency in the main working section.

\subsubsection{Basic Parameters of Target Automobile}

Combined with the project requirements, this paper takes a small electric automobile as the research object and the vehicle parameter and dynamic performance indexes of the automobile are shown in Table 1. 
Designing a New Type of Electric Machinery Continuously Variable Transmission Combined with Matia/Simulink Simulation Kinematics Model Based on CATIA and ADAMS Monitoring and Management Software System of Oil Pipe Hydraulic Press

Table 1: the vehicle parameters of the target vehicle

\begin{tabular}{|c|c|c|c|}
\hline \multirow{4}{*}{$\begin{array}{c}\text { Date of } \\
\text { complete } \\
\text { Motorcycle }\end{array}$} & $\begin{array}{l}\text { No-load } \\
\text { quality }\end{array}$ & $\begin{array}{c}\text { Full load } \\
\text { quality }\end{array}$ & $\begin{array}{c}\text { Windward } \\
\text { area }\end{array}$ \\
\hline & 800 & 1000 & 1.8 \\
\hline & \begin{tabular}{l}
\multicolumn{1}{c}{ Air } \\
resistance \\
coefficient
\end{tabular} & $\begin{array}{l}\text { Rolling } \\
\text { resistance } \\
\text { coefficient }\end{array}$ & $\begin{array}{l}\text { Wheel } \\
\text { radius }\end{array}$ \\
\hline & 0.3 & 0.015 & 0.31 \\
\hline \multirow{2}{*}{$\begin{array}{c}\text { Dynamic } \\
\text { performance }\end{array}$} & $\begin{array}{l}\text { Maximum } \\
\text { speed }\end{array}$ & & Gradient \\
\hline & 100 & & $30 \%$ \\
\hline
\end{tabular}

The force of the electric automobile is analyzed based on Table 1. Firstly, the external forces received by the electric automobile during the driving process are mainly divided into two types: the first is the driving force, which is provided by the motor to generate the kinetic energy and potential energy required by the motion; the other type is the resistance imposed by the outside world during the driving process. This includes wind resistance, roll resistance, slope resistance and resistance of acceleration.

Under this premise, this paper mainly uses the permanent magnet synchronous motor for vehicles as the designed main motor and the permanent magnet synchronous motor can switch between the two states of electric motion and power generation corresponding to different driving conditions of the automobile. During normal driving, the chemical energy in the power battery pack is converted into mechanical energy output to the traveling mechanism through the motor; when braking or coasting, the motor enters the power generation mode and the mechanical energy of the system is converted into the electric energy to store in battery packs. The working principle is shown in Figure 5.

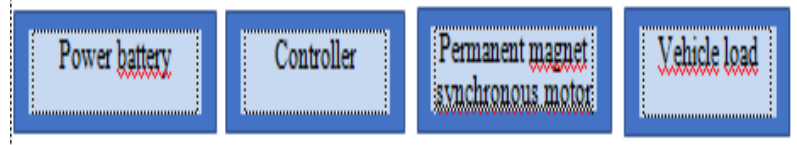

Figure 5: working principle of permanent magnet synchronous motor

\subsubsection{Design of transmission scheme}

According to the analysis of planetary gear theory, it can be seen that the multi-stage planetary gear set formed by the series connection of various one-part planetary gears can better expand the range of speed regulation of the transmission system, but the combination of various single gears will inevitably increase the complexity of the planetary gear and significantly increase the mechanical cost and weight.
The purpose of this paper is to study the characteristics of the electric machinery continuous transmission, and only small corresponding test bench is prepared in the follow-up test while the reverse gear is not considered for the time being. Due to the limitation of the experimental cost, the two-stage planetary gear set is used.

\subsection{Parameter optimization design of electric machinery continuously variable transmission}

The transmission structural parameter directly determines its transmission efficiency and speed regulation performance and this optimization is multivariable nonlinear optimization, which is very difficult to solve using some conventional optimization methods such as the fastest descent method and gradient method. In recent years, intelligent optimization algorithms have been developing continuously and algorithms such as neural networks, genetic algorithms, ant colony algorithms, particle swarm optimization, tabu search, and hybrid optimization strategies have made great progress in both theoretical research and practical applications, whose theories include mathematics, physics, biology, artificial intelligence, neuroscience and statistics.

The development and research of these algorithms have made it possible to solve complicated and computationally intensive optimization problems. This paper mainly uses the particle swarm algorithm to for calculation.

The calculation result is: Matlab software has various built-in programs of optimization algorithms, and the corresponding toolbox can be directly called, including the particle swarm algorithm (PSOt). The PSOt is very practical. In the calculation, it is only necessary to obtain the script file corresponding to the objective function and constraint conditions according to the analysis in the last chapter and then call the corresponding program of the particle swarm algorithm through the command.

\section{Results and Analysis}

\subsection{Simulation results and analysis}

Without considering the acceleration compensation torque, only the electric vehicle is driven by the constant speed driving torque at a constant speed and the accelerator pedal opening changes from 0 to 1 in the three work sections respectively. At this time, for the two motors in the starting section, the system efficiency of increases gradually with the vehicle speed. When the speed increases to $V=20 \mathrm{~km} / \mathrm{h}$, it can be increased to $0.859 \mathrm{~F}$; at the beginning of Stage II $(\mathrm{v}<45.56 \mathrm{~km} / \mathrm{h})$, due to the M2 power split ratio $\mathrm{p}<0$, the internal 
Designing a New Type of Electric Machinery Continuously Variable Transmission Combined with Matia/Simulink Simulation Kinematics Model Based on CATIA and ADAMS Monitoring and Management Software System of Oil Pipe Hydraulic Press

transmission efficiency is relatively lower. However, in the second half $(\mathrm{v}>45.56 \mathrm{~km} / \mathrm{h}) \mathrm{p}>0$, the efficiency increase is rather significant with the maximum of 0.892; in section I, because there is no power cycle interval range $\mathrm{p}<0$, the overall transmission efficiency is relatively high, up to 0.936 . The above analysis reflects the overall efficiency of the transmission system. Since the highest efficiency of the motor itself can only reach $95 \%$, the overall transmission efficiency is calculated on the basis of the motor efficiency. It can be seen that the electric machinery continuously variable transmission designed in this paper can maintain relatively good efficiency characteristics when driven at a constant speed under standard road conditions.

\subsection{Analysis of acceleration performance}

The simulation verification is conducted on the acceleration performance of the designed transmission system under standard road conditions. In the three sections of I, II and III, the accelerator pedal opening $6>\mathrm{F}=\mathrm{F} 0$ is taken as the starting point. The signal is applied to the pedal and the opening reaches its maximum at $2 \mathrm{~s}$. Under a given acceleration signal, the speed of the electric vehicle increases from $45.56 \mathrm{~km} / \mathrm{h}$ to $100 \mathrm{~km} / \mathrm{h}$ in8.86s in section I.

\section{Conclusion}

The continuously variable transmission technology can complete the optimal match between the vehicle and the driving equipment and has a significant role in enhancing the dynamics and economy of the vehicle. In current stage of energy constraint and increasing environmental protection requirements, the electric vehicle will become the main research direction of the automotive industry.

Aiming at the continuously variable transmission technology applied in electric vehicles, this paper designs the corresponding electric machinery continuously variable transmission.

An electric mechanical continuously variable transmission is proposed combined with the planetary transmission theory and six schemes of the transmission mode of the model are studied. The characteristics of various transmission schemes are analyzed, including speed, power flow direction and efficiency characteristics; the characteristics of the two-stage planetary transmission is analyzed based on the one-part planetary gear and the transmission scheme suitable for the electric machinery continuously variable transmission is selected through the study of characteristics.

The electric machinery continuously variable transmission test stand is established and the stage test under the steady working condition is conducted on the continuously variable transmission designed in this paper. The operation data is collected and analyzed to verify that the electric mechanical continuously variable transmission has a stable continuously variable speed regulation characteristics and relatively high system efficiency characteristics within its speed regulation range.

\section{References}

[1] Cheng M, Sun L, Buja G and Song L, "Advanced electrical machines and machine-based systems for electric and hybrid vehicles", Energies, 2015, Volume 8, Issue 9, Pages 9541-9564. DOI: 10.3390/en8099541

[2] Druant J, De Belie F, Sergeant P and Melkebeek J, "Field-oriented control for an induction-machinebased electrical variable transmission", IEEE Transactions on Vehicular Technology, 2016, Volume 65, Issue 6, Pages 4230-4240. DOI: 10.1109/TVT.2015.2496625

[3] Liu Y, Niu S and Fu W, "Design of an electrical continuously variable transmission based wind energy conversion system", IEEE Transactions on Industrial Electronics, 2016, Volume 63, Issue 11, Pages 6745-6755. DOI: 10.1109/TIE.2016.2590383

[4] Petković D, Ćojbašić Ž, Nikolić V, Shamshirband S, Kiah MLM, Anuar NB and Wahab AWA, "Adaptive neuro-fuzzy maximal power extraction of wind turbine with continuously variable transmission", Energy, 2014, Volume 64, Pages 868-874. DOI: 10.1016/j.energy.2013.10.094

[5] Shamshirband S, Petković D, Amini A, Anuar NB, Nikolić V, Ćojbašić Ž and Gani A, "Support vector regression methodology for wind turbine reaction torque prediction with power-split hydrostatic continuous variable transmission", Energy, 2014, Volume 67, Pages 623-630. DOI: 10.1016/j.energy.2014.01.111

[6] Sulaiman N, Hannan MA, Mohamed A, Majlan EH and Daud WW, "A review on energy management system for fuel cell hybrid electric vehicle: Issues and challenges", Renewable and Sustainable Energy Reviews, 2015, Volume 52, Pages 802814. DOI: $10.1016 /$ j.rser.2015.07.132

[7] Tyreas GC and Nikolakopoulos PG, "Development and friction estimation of the Half-Toroidal Continuously Variable Transmission: A wind generator application", Simulation Modelling Practice and Theory, 2016, Volume 66, Pages 6380. DOI: 10.1016/j.simpat.2015.11.007

[8] Yang Y, Schofield N and Emadi A, "Integrated electromechanical double-rotor compound hybrid transmissions for hybrid electric vehicles", IEEE Transactions on Vehicular Technology, 2016, Volume 65, Issue 6, Pages 4687-4699. DOI: 10.1109/TVT.2015.2487301 